\author{
Glenda Blaser Petarlia \\ (D) http://orcid.org/0000-0002-6828-1238 \\ Monica Cattafesta ${ }^{a}$ \\ (iD) https://orcid.org/0000-0002-8973-622X \\ Tamires Conceição da Luz ${ }^{\mathrm{a}}$ \\ (D) https://orcid.org/0000-0003-1771-2998 \\ Eliana Zandonade ${ }^{a}$ \\ (iD) https://orcid.org/0000-0001-5160-3280 \\ Olívia Maria de Paula Alves Bezerrab \\ (iD) https://orcid.org/0000-0002-5596-657X \\ Luciane Bresciani Salaroli ${ }^{a}$ \\ (iD) https://orcid.org/0000-0002-1881-0306
}

anniversidade Federal do Espírito Santo (UFES), Centro de Ciências da Saúde. Vitória, ES, Brasil.

bUniversidade Federal de Ouro Preto (UFOP), Escola de Medicina, Departamento de Medicina de Família, Saúde Mental e Coletiva. Ouro Preto, MG, Brasil.

Contato:

Glenda Blaser Petarli

E-mail

glenda.petarli@gmail.com

Os autores informam que o estudo original do qual foram derivados os dados deste artigo foi financiado pela Fundação de Amparo à Pesquisa do Espírito Santo (Fapes) - Edital Fapes/ CNPq/Decit-SCTIE-MS/SESA - PPSUS $n^{\circ} 05 / 2015$.

Os autores declaram que não há conflitos de interesses.

Os autores informam que o trabalho não foi apresentado em eventos científicos

O trabalho é oriundo da pesquisa de doutorado de Glenda Blaser Petarli intitulada Saúde de agricultores do Espírito Santo: a complexa relação entre produção de alimentos, exposição a agrotóxicose risco à saúde humana, apresentada ao Programa de Pós-Graduação em Saúde Coletiva da Universidade Federal do Espírito Santo em 2019.

Recebido: 01/07/2018

Revisado: 27/10/2018

Aprovado: 07/12/2018

\section{Exposição ocupacional a agrotóxicos, riscos e práticas de segurança na agricultura familiar em município do estado do Espírito Santo, Brasil}

\author{
Occupational exposure to pesticides, risks and safety practices \\ in family farming in a municipality of Espírito Santo, Brazil
}

\section{Resumo}

Objetivo: caracterizar a exposição ocupacional, percepção do risco, práticas de segurança e fatores associados ao uso de equipamento de proteção individual (EPI) durante a manipulação de agrotóxicos. Métodos: estudo transversal com amostra representativa de agricultores de Santa Maria de Jetibá, Espírito Santo. Procedeu-se à caracterização sociodemográfica e ocupacional dos agricultores com exposição direta a agrotóxicos e a identificação dos ingredientes ativos e classificação toxicológica dos produtos utilizados. Resultados: foram referidas 106 marcas comerciais, 45 grupos químicos e 77 ingredientes ativos. Houve predomínio do herbicida glifosato. Dos 550 agricultores avaliados 89\% referiram uso de agrotóxicos extremamente tóxicos, 56,3\% utilizavam mais de cinco agrotóxicos e 51\% trabalhavam há mais de 20 anos em contato direto com estes produtos. Metade não lia rótulo dos agrotóxicos, mais de um terço não observava o tempo de carência para colheita e reaplicação e nem o de reentrada na lavoura; 71,4\% não utilizavam EPI ou utilizavam de forma incompleta. Entre os fatores associados à não utilização do EPI, destaca-se a classe socioeconômica $(p=0,002)$, baixa escolaridade $(p=0,05)$, falta de suporte técnico $(\mathrm{p}<0,001)$ e não leitura dos rótulos $(\mathrm{p}<0,001)$. Conclusão: os agricultores apresentaram exposição ocupacional prolongada a múltiplos agrotóxicos de elevada toxidade, referindo práticas inseguras de manuseio.

Palavras-chave: agrotóxicos; agricultura familiar; trabalho rural; exposição ocupacional; saúde do trabalhador.

\begin{abstract}
Objective: to characterize occupational exposure, risk perception, safety practices and factors associated with the use of personal protective equipment (PPE) during pesticide handling. Methods: cross-sectional study with a representative sample of farmers from Santa Maria de Jetibá, Espírito Santo, Brazil. We collected sociodemographic and occupational data of farmers directly exposed to pesticides and identified the active ingredients and toxicological classification of the pesticides used. Results: 106 trademarks, 45 chemical groups and 77 active ingredients were identified. There was a predominance of the herbicide glyphosate. Of the 550 farmers evaluated, 89\% reported using extremely toxic pesticides, $56.3 \%$ used more than five different agrochemicals, and 51\% worked for over 20 years in direct contact with these products. Half of them did not read the label, more than a third did not observe the pre-harvest, reapplication, or reentry intervals; $71.4 \%$ used incomplete PPE or none. Factors associated with non-use of PPE include socioeconomic class $(p=0.002)$, low educational level $(p=0.05)$, lack of technical support $(p<0.001)$ and non-reading of labels $(p<0.001)$. Conclusion: farmers have prolonged occupational exposure to multiple high toxicity pesticides, referring to unsafe handling practices.
\end{abstract}

Keywords: pesticides; family farming; rural work; occupational exposure; occupational health. 


\section{Introdução}

Considerado um importante fator para o aumento da produtividade das lavouras, a utilização de agrotóxicos tornou-se parte integrante do cotidiano agrícola. No entanto, a exposição humana a essas substâncias representa um problema de saúde pública ${ }^{1}$, visto que seu uso intensivo e indiscriminado tem acarretado consequências negativas para o ambiente e para a saúde das populações ${ }^{2}$. Em 2016, cerca de 550 mil toneladas desses produtos foram vendidos no Brasil ${ }^{3}$, que desde 2008 ocupa a posição de maior consumidor mundial de agrotóxicos ${ }^{1}$.

Devido a sua toxicidade intrínseca, os agrotóxicos impactam a saúde humana, produzindo efeitos que variam conforme o princípio ativo, a dose absorvida e a forma de exposição ${ }^{1}$. Além disso, a ampla utilização desses produtos, o desconhecimento dos riscos associados a sua utilização, o desrespeito às normas de segurança em seu manuseio, a livre comercialização, a grande pressão comercial por parte das empresas distribuidoras e produtoras e os problemas sociais encontrados no meio rural constituem importantes causas que levam ao agravamento dos quadros de contaminação humana e ambiental observados no Brasil ${ }^{4}$.

Cabe destacar que, a partir do conhecimento das lavouras predominantes, dos tipos de agrotóxicos utilizados e de suas características toxicológicas, pode-se inferir as previsões de contaminação ambiental e intoxicações humanas em cada região, servindo de alerta aos profissionais de saúde para subsidiar as ações de Vigilância em Saúde ${ }^{5}$.

Visando contribuir para o planejamento de políticas públicas voltadas à vigilância das populações expostas a agrotóxicos, este estudo teve o objetivo de caracterizar a exposição ocupacional a agrotóxicos, percepção do risco, práticas de segurança e fatores associados ao uso de equipamento de proteção individual (EPI) durante a manipulação de agrotóxicos em agricultores de Santa Maria de Jetibá, considerado o principal município agrícola do Espírito Santo.

\section{Metodologia}

Trata-se de estudo epidemiológico descritivo quantitativo derivado de projeto mais amplo, de base populacional, intitulado "Condição de saúde e fatores associados: um estudo em agricultores do Espírito Santo" (AgroSaúdES), financiado pela Fundação de Amparo à Pesquisa do Espírito Santo (Fapes) e, desenvolvido no município de Santa Maria de Jetibá, região serrana do Espírito Santo, Brasil.

O AgroSaúdES envolveu amostra representativa de agricultores de ambos os sexos que atendiam aos seguintes critérios de inclusão: indivíduos de 18 a 59 anos, não gestantes, que tivessem a agricultura como principal fonte de renda e estivessem em plena atividade laboral por, no mínimo, seis meses. A identificação dos participantes foi realizada por meio dos dados disponíveis nos cadastros de indivíduos e de famílias realizado pelas equipes da Estratégia Saúde da Família, responsáveis pela cobertura de $100 \%$ das 11 regiões de saúde do município.

Foram identificados 7.287 agricultores de um total de 4.018 famílias. O cálculo do tamanho amostral considerou prevalência de desfecho (utilização de EPI) de $50 \%{ }^{6}$, erro amostral de $3,5 \%$ e nível de confiança de 95\%, perfazendo uma amostra mínima de 708 pessoas. Para compensar possíveis perdas, foram convidados 806 agricultores, cuja seleção ocorreu mediante sorteio estratificado considerando o número de famílias por região de saúde e por agente comunitário de saúde (ACS), de modo a respeitar a proporcionalidade entre as 11 regiões e entre os 80 ACS. Admitiu-se apenas um indivíduo sorteado por família, evitando assim a interdependência de informações. Em caso de recusa ou não comparecimento, foi convocado um novo participante de uma lista reserva do sorteio, respeitando-se o sexo e a unidade de saúde de origem do desistente.

Cabe destacar que, devido às características do município de Santa Maria de Jetibá, onde predomina a agricultura familiar, participaram do estudo apenas pequenos agricultores, cujas práticas agrícolas caracterizam-se pelo predomínio da policultura e baixo grau de mecanização.

A coleta de dados ocorreu entre dezembro de 2016 e abril de 2017 nas dependências das unidades de saúde do município. Foi aplicado questionário semiestruturado contendo questões sobre características socioeconômicas, demográficas, laborais e de exposição ocupacional a agrotóxicos, incluindo os produtos utilizados, frequência de uso, percepção de risco e práticas adotadas durante a manipulação de agrotóxicos.

Foram excluídos participantes que não apresentavam exposição ocupacional direta a agrotóxicos, considerada como a manipulação em qualquer uma das etapas de armazenamento, transporte, preparo, aplicação, descarte, e descontaminação de equipamentos e vestimenta. 
As variáveis sociodemográficas analisadas foram: sexo, faixa etária, escolaridade, estado civil e classe socioeconômica. Esta última foi determinada de acordo com o Critério de Classificação Econômica Brasil (Abep) ${ }^{7}$.

Para a caracterização ocupacional, considerou-se o tempo de trabalho como agricultor, a carga horária semanal de trabalho na agricultura, a posse/propriedade da terra, o total de culturas produzidas e os principais tipos de cultivos.

Para caracterização do uso e das práticas de segurança adotadas para manipulação de agrotóxicos, avaliou-se: a marca comercial, o número de produtos citados, o tempo (anos) de exposição ocupacional, o período decorrido desde o último contato, se houve orientação técnica para a aquisição dos produtos, o tipo de equipamento utilizado para aplicação, a utilização de EPI, o hábito de leitura do rótulo das embalagens e a observância do tempo estabelecido no rótulo e/ou no receituário agronômico para reentrada na lavoura e tempo de carência para a reaplicação ou colheita dos alimentos após aplicação.

A aquisição dos agrotóxicos foi considerada "Com orientação técnica" quando a indicação era realizada por profissionais da Assistência Técnica e Extensão Rural (Ater) do município e "Sem orientação técnica" quando tal indicação era realizada por vizinhos, vendedores ou por iniciativa própria.

A utilização de EPI foi categorizada em "EPI completo", "EPI incompleto" e "Não utiliza EPI". Foi considerado "EPI completo" o uso no, momento do contato com agrotóxicos, dos seguintes itens: touca, jaleco e calça de materiais resistentes a produtos químicos, óculos de proteção, máscaras/respiradores, luvas e botas impermeáveis.

Para caracterização química e toxicológica dos agrotóxicos empregados, foi utilizado o Sistema de Agrotóxicos Fitossanitários (Agrofit) ${ }^{8}$, identificando-se grupo químico, ingrediente ativo, classificação toxicológica e de periculosidade ambiental, utilizando-se como termos de busca as marcas comerciais dos produtos.

A percepção dos riscos referentes aos agrotóxicos foi avaliada por meio das perguntas: "O(a) sr.(a) acha que os agrotóxicos podem fazer mal para saúde do agricultor?" e "O(a) sr.(a) acha que os agrotóxicos podem fazer mal para saúde das pessoas que consomem os alimentos produzidos com agrotóxicos?", admitindo-se com resposta "sim", "não" e "não sei".

Para análise descritiva dos dados, foram calculados os valores absolutos e percentuais de cada variável. Empregou-se o teste qui-quadrado para determinação dos fatores associadas à utilização de EPIs, adotando-se nível de significância de 5\%. Foram apresentadas na tabela apenas as variáveis com associação estatisticamente significante. Utilizou-se para as análises o programa IBM SPSS Statistics 22.0.

O estudo original foi aprovado pelo Comitê de Ética em Pesquisa do Centro de Ciências de Saúde da Universidade Federal do Espírito Santo, Parecer $\mathrm{n}^{\circ} 2091172$ (CAAE 52839116.3.0000.5060). Todos os participantes assinaram o Termo de Consentimento Livre e Esclarecido (TCLE).

\section{Resultados}

Dos 806 agricultores convidados para o estudo, foram efetivamente avaliados 790. Destes, foram considerados para fins deste artigo os dados de 550 $(69,62 \%)$ por referirem contato ocupacional direto com agrotóxicos. Neste grupo (Tabela 1), houve predomínio de indivíduos do sexo masculino, faixa etária entre 30 a 39 anos e com menos de quatro anos de estudo. Mais de $80 \%$ deles se declararam casados ou vivendo com companheiro(a). A classe socioeconômica $\mathrm{C}$ foi predominante.

A caracterização ocupacional expressa na Tabela 2 mostra que $44,9 \%$ dos agricultores trabalhavam na atividade há mais de 30 anos, 84,2\% relataram jornada de trabalho superior a 40 horas semanais e $77,8 \%$ referiram serem proprietários da terra em que trabalhavam. Houve predomínio de cultivo de 5 a 10 tipos de culturas, sendo, em sua maior parte, lavouras temporárias. Os cultivos mais citados foram repolho, feijão e milho.

Com relação ao uso e às práticas de segurança na manipulação dos agrotóxicos (Tabela 3) verificou-se que a maior parte dos agricultores utiliza habitualmente mais de cinco tipos diferentes de agrotóxicos e está há mais de 20 anos trabalhando em contato direto com esses produtos. Mais de 60\% relataram ter tido contato ocupacional com agrotóxicos nos sete dias anteriores à entrevista. Uma parcela expressiva relatou comprar os produtos sem orientação técnica da Ater, utilizar pulverizador costal manual, e apenas 28,6\% relataram utilizar EPI completo. O motivo mais citado para não utilização de EPI foi o desconforto. Aproximadamente metade dos agricultores relatou não ler o rótulo dos agrotóxicos; $35,5 \%$ referiram não observar o tempo de carência para colheita, 32\% não observavam o período de carência para reaplicação e $46,2 \%$ não cumpriam o período de reentrada na lavoura. Quando questionados sobre a percepção dos riscos referentes à utilização de agrotóxicos, 94,7\% referiram considerar que esses produtos fazem mal à saúde do agricultor e $74,9 \%$ consideraram que ocasionam efeitos nocivos à saúde de quem consome os alimentos. 
Tabela 1 Caracterização sociodemográfica de agricultores do município de Santa Maria de Jetibá, ES

\begin{tabular}{|c|c|c|c|}
\hline Variáveis & $n$ & $\%$ & $I_{95 \%}$ \\
\hline \multicolumn{4}{|l|}{ Sexo } \\
\hline Masculino & 355 & 64,5 & $61-69$ \\
\hline Feminino & 195 & 34,5 & $31-39$ \\
\hline \multicolumn{4}{|l|}{ Faixa Etária } \\
\hline Até 29 anos & 143 & 26,0 & $22-30$ \\
\hline 30 a 39 anos & 166 & 30,2 & $26-34$ \\
\hline 40 a 49 anos & 139 & 25,3 & $21-28$ \\
\hline 50 anos ou mais & 102 & 18,5 & $16-22$ \\
\hline \multicolumn{4}{|l|}{ Escolaridade } \\
\hline Menos de 4 anos & 374 & 68,0 & $64-72$ \\
\hline 4 a 8 anos & 122 & 22,2 & $19-26$ \\
\hline Mais de 8 anos & 54 & 9,8 & $7-12$ \\
\hline \multicolumn{4}{|l|}{ Estado Civil } \\
\hline Solteiro & 45 & 8,2 & $6-10$ \\
\hline Casado(a)/Vive com companheiro(a) & 464 & 84,4 & $81-87$ \\
\hline Separado(a)/Divorciado(a)/Viúvo(a) & 41 & 7,5 & $5-10$ \\
\hline \multicolumn{4}{|l|}{ Classe Socioeconômica* } \\
\hline Classe A ou B & 42 & 7,6 & $5-10$ \\
\hline Classe C & 279 & 50,7 & $46-55$ \\
\hline Classe D ou E & 229 & 41,6 & $37-46$ \\
\hline
\end{tabular}

$\mathrm{IC}_{95 \%}$ : intervalo de $95 \%$ de confiança.

*Baseada no Critério de Classificação Econômica Brasil (Abep).

Tabela 2 Caracterização ocupacional de agricultores do município de Santa Maria de Jetibá, ES

\begin{tabular}{|c|c|c|c|}
\hline Variáveis & $n^{*}$ & $\%$ & $I C_{95 \%}$ \\
\hline \multicolumn{4}{|l|}{ Tempo de trabalho como agricultor } \\
\hline Menos de 10 anos & 24 & 4,4 & $3-6$ \\
\hline De 10 a 29 anos & 278 & 50,7 & $46-55$ \\
\hline 30 anos ou mais & 246 & 44,9 & $41-49$ \\
\hline \multicolumn{4}{|l|}{ Carga horária semanal de trabalho } \\
\hline Menor ou igual a 40 horas & 87 & 15,8 & $13-19$ \\
\hline Maior de 40 horas & 463 & 84,2 & $81-87$ \\
\hline \multicolumn{4}{|l|}{ Posse/Propriedade da Terra } \\
\hline Proprietário & 428 & 77,8 & $74-81$ \\
\hline Não-proprietário & 122 & 22,2 & $19-26$ \\
\hline \multicolumn{4}{|l|}{ Total de culturas cultivadas } \\
\hline Até 4 culturas & 245 & 44,5 & $40-49$ \\
\hline 5 a 10 culturas & 263 & 47,8 & $43-52$ \\
\hline 11 ou mais culturas & 42 & 7,6 & $5-10$ \\
\hline \multicolumn{4}{|l|}{ Tipo de lavoura predominante } \\
\hline Lavoura somente temporária & 279 & 50,7 & $46-55$ \\
\hline Lavoura somente permanente & 43 & 7,8 & $5-10$ \\
\hline Lavoura temporária e permanente & 228 & 41,5 & $37-45$ \\
\hline
\end{tabular}

(Continua) 
Tabela 2 Continuação...

\begin{tabular}{llll}
\hline & Variáveis & $n^{*}$ & $I_{95 \%}$ \\
\hline Culturas cultivadas*** & & & $47-55$ \\
Repolho & 282 & 47,2 & $43-51$ \\
Feijão & 259 & 43,1 & $39-47$ \\
Milho & 237 & 29,4 & $25-33$ \\
Café & 162 & 28,3 & $24-32$ \\
Inhame & 156 & $23-30$ \\
Pimentão & 146 & $23-27$ \\
Abobrinha & 131 & 23,8 & $19-26$ \\
Morango & 127 & 23,1 & $18-25$ \\
Chuchu & 121 & 22,0 & $18-25$ \\
Couve-flor & 121 & 22,0 & $18-25$ \\
Vagem & 119 & 21,6 & $18-25$ \\
Beterraba & 118 & 21,4 & $17-23$ \\
Tomate & 110 & 20,0 & 25 \\
\hline
\end{tabular}

$\mathrm{IC}_{95 \%}$ : intervalo de $95 \%$ de confiança.

*n variou de 548 a 550 em função de não respostas. *****am apresentados os dados das 13 culturas citadas com maior frequência; no total,

59 tipos de culturas foram relatadas.

Tabela 3 Caracterização da exposição ocupacional, práticas de manipulação e percepção do risco para saúde relacionado à manipulação de agrotóxicos pelos agricultores do município de Santa Maria de Jetibá, ES

\begin{tabular}{|c|c|c|c|}
\hline Variáveis & $n^{*}$ & $\%$ & $I C_{95 \%}$ \\
\hline \multicolumn{4}{|l|}{ Número de agrotóxicos utilizados } \\
\hline Até 5 tipos de agrotóxicos & 223 & 43,7 & $39-48$ \\
\hline Mais de 5 agrotóxicos & 287 & 56,3 & $52-60$ \\
\hline \multicolumn{4}{|l|}{ Anos de trabalho com agrotóxicos } \\
\hline 20 anos ou menos & 263 & 49,0 & $45-53$ \\
\hline Mais de 20 anos & 274 & 51,0 & $47-55$ \\
\hline \multicolumn{4}{|l|}{ Tempo desde o último contato } \\
\hline Nos últimos 7 dias & 335 & 61,6 & $57-65$ \\
\hline Mais de 7 dias & 209 & 38,4 & $34-42$ \\
\hline \multicolumn{4}{|l|}{ Orientação técnica para aquisição de Agrotóxicos } \\
\hline Não é responsável pela compra de agrotóxicos & 184 & 33,5 & $29-37$ \\
\hline Aquisição sem orientação técnica da Ater & 188 & 34,2 & $30-38$ \\
\hline Aquisição com orientação técnica da Ater & 178 & 32,4 & $28-36$ \\
\hline \multicolumn{4}{|l|}{ Equipamento de aplicação } \\
\hline Pulverizador Costal Manual & 302 & 59,3 & $55-63$ \\
\hline Pulverizador Costal Mecanizado & 102 & 20,0 & $16-23$ \\
\hline Utiliza ambas as formas de aplicação & 105 & 20,6 & $17-24$ \\
\hline \multicolumn{4}{|l|}{ Conduta após aplicar agrotóxicos } \\
\hline Toma banho imediatamente & 450 & 84,7 & $81-88$ \\
\hline Apenas se lava imediatamente & 32 & 6,0 & $4-8$ \\
\hline Toma banho algumas horas após a aplicação & 37 & 7,0 & $4-9$ \\
\hline Lava-se algumas horas após a aplicação & 2 & 0,4 & $0,1-0,9$ \\
\hline Não toma banho ou não se lava após aplicação & 10 & 1,9 & $0,7-3$ \\
\hline
\end{tabular}

(Continua) 
Tabela 3 Continuação...

\begin{tabular}{|c|c|c|c|}
\hline Variáveis & $n^{*}$ & $\%$ & $I C_{95 \%}$ \\
\hline \multicolumn{4}{|l|}{ Utilização de EPI橉 } \\
\hline Utiliza EPI completo & 152 & 28,6 & $25-32$ \\
\hline Utiliza EPI incompleto & 259 & 48,7 & $44-53$ \\
\hline Não utiliza EPI & 121 & 22,7 & $19-26$ \\
\hline \multicolumn{4}{|l|}{ Motivo para não utilização do EPI ${ }^{* * *}$} \\
\hline Por considerá-los desconfortáveis & 48 & 41,4 & $32-50$ \\
\hline Por não considerar necessário utilizar & 38 & 32,8 & $24-41$ \\
\hline Por considerá-los de alto custo & 23 & 19,8 & $12-27$ \\
\hline Por não saber como utilizar & 7 & 6,0 & $1,7-10$ \\
\hline \multicolumn{4}{|l|}{ Realizada leitura do rótulo dos agrotóxicos } \\
\hline Sim & 260 & 50,6 & $46-55$ \\
\hline Não & 254 & 49,4 & $45-54$ \\
\hline \multicolumn{4}{|l|}{ Respeita o tempo de carência para colheita } \\
\hline Sim & 330 & 64,5 & $60-68$ \\
\hline Não & 182 & 35,5 & $31-39$ \\
\hline \multicolumn{4}{|c|}{ Respeita o tempo de carência para reaplicação } \\
\hline Sim & 340 & 68,0 & $64-72$ \\
\hline Não & 160 & 32,0 & $28-36$ \\
\hline \multicolumn{4}{|c|}{ Respeita o tempo de reentrada na lavoura após aplicação } \\
\hline Sim & 278 & 53,8 & $49-58$ \\
\hline Não & 239 & 46,2 & $42-50$ \\
\hline \multicolumn{4}{|c|}{ Considera o agrotóxico prejudicial à saúde do agricultor } \\
\hline Sim & 521 & 94,7 & $93-96$ \\
\hline Não & 20 & 3,6 & $2-5$ \\
\hline Não sabe & 9 & 1,6 & $0,5-2,7$ \\
\hline \multicolumn{4}{|c|}{ Considera o agrotóxico prejudicial à saúde do consumidor } \\
\hline Sim & 412 & 74,9 & $71-78$ \\
\hline Não & 106 & 19,3 & $16-22$ \\
\hline Não sabe & 32 & 5,8 & $4-8$ \\
\hline
\end{tabular}

$\mathrm{IC}_{95 \%}$ : Intervalo de 95\% de confiança; Ater: Assistência Técnica e Extensão Rural; EPI: equipamento de proteção individual.

*n variou de 500 a 550 em função de não respostas. **** EPI: equipamento de proteção individual

Conforme apresentado na Tabela 4, estiveram associados à não utilização de EPI o sexo feminino ( $p<0,001)$, baixa escolaridade $(p=0,05)$, baixa classe socioeconômica $(p=0,002)$, cultivar lavouras temporárias $(\mathrm{p}=0,023)$, utilizar até 5 agrotóxicos $(0,044)$, comprar agrotóxicos sem orientação técnica ( $\mathrm{p}<0,001)$, utilizar equipamento não mecanizado para aplicação $(p=0,002)$, não tomar banho ou se lavar adequadamente após o uso $(p<0,001)$ e não observar os tempos de carência para reentrada ( $p<0,001)$, reaplicação $(p<0,001)$ e colheita dos produtos após aplicar agrotóxicos $(\mathrm{p}<0,001)$.

Foram citadas 106 marcas comerciais diferentes de agrotóxicos, totalizando 45 grupos químicos e 77 ingredientes ativos distintos. Com relação à marca comercial, o herbicida Roundup ${ }^{\circledR}$ foi o produto mais citado, seguido pelo Gramoxone ${ }^{\circledR}$, pelo inseticida Decis ${ }^{\circledR}$ e pelo fungicida Dithane $\mathrm{Nt}^{\circledR}$. Analisando-se o ingrediente ativo (Tabela 5), o glifosato sal di-amônio foi o mais prevalente, sendo utilizado por $66,4 \%$ dos agricultores, seguido pelo fungicida mancozebe, o herbicida dicloreto de paraquate e em, quarto lugar, o inseticida deltametrina.

Com relação à finalidade, herbicidas foram mais frequentes, utilizados por 78,8\% dos agricultores. Analisando-se a classe toxicológica, $88,8 \%$ dos agricultores utilizavam agrotóxicos "extremamente tóxicos à saúde humana" e mais de $91 \%$ produtos classificados como "muito perigoso" para o meio ambiente $^{\mathrm{c}}$.

c A classificação toxicológica utilizada para este estudo foi baseada na normativa vigente à época (Portaria Ms/Snvs, $n^{\circ} 3$, de 16 de janeiro de 1992). Cabe destacar que os critérios de classificação foram posteriormente alterados pela Resolução DC/Anvisa $\mathrm{n}^{\circ} 294$ de 29 de julho de 2019, publicada no Diário Oficial da União em 31/07/2019. 
Tabela 4 Variáveis associadas à utilização de equipamento de proteção individual por agricultores de Santa Maria de Jetibá, ES

\begin{tabular}{|c|c|c|c|c|c|c|c|}
\hline \multirow{2}{*}{ Variáveis } & \multicolumn{2}{|c|}{ EPI*completo } & \multicolumn{2}{|c|}{ EPI*incompleto } & \multicolumn{2}{|c|}{ Não utiliza EPI* } & \multirow[b]{2}{*}{ p-valor } \\
\hline & $n$ & $\%$ & $n$ & $\%$ & $n$ & $\%$ & \\
\hline \multicolumn{8}{|l|}{ Sexo } \\
\hline Masculino & 115 & 32,5 & 183 & 51,7 & 56 & 15,8 & $<0,001$ \\
\hline Feminino & 37 & 20,8 & 76 & 42,7 & 65 & 36,5 & \\
\hline \multicolumn{8}{|l|}{ Escolaridade } \\
\hline Menos de 4 anos & 110 & 30,6 & 161 & 44,7 & 89 & 24,7 & 0,050 \\
\hline 4 a 8 anos & 27 & 22,7 & 66 & 55,5 & 26 & 21,8 & \\
\hline Mais de 8 anos & 15 & 28,3 & 32 & 60,4 & 6 & 11,3 & \\
\hline \multicolumn{8}{|l|}{ Classe Socioeconômica**** } \\
\hline Classe A ou B & 12 & 28,6 & 22 & 52,4 & 8 & 19,0 & 0,002 \\
\hline Classe C & 81 & 30,2 & 144 & 53,7 & 43 & 16,0 & \\
\hline Classe D ou E & 59 & 26,6 & 93 & 41,9 & 70 & 31,5 & \\
\hline \multicolumn{8}{|l|}{ Tipo de lavoura predominante } \\
\hline Lavoura somente temporária & 71 & 26,5 & 120 & 44,8 & 77 & 28,7 & 0,023 \\
\hline Lavoura somente permanente & 13 & 31,7 & 20 & 48,8 & 8 & 19,5 & \\
\hline Lavoura temporária e permanente & 68 & 30,5 & 119 & 53,4 & 36 & 16,1 & \\
\hline \multicolumn{8}{|l|}{ Número de agrotóxicos utilizados } \\
\hline Até 5 tipos de agrotóxicos & 65 & 30,5 & 90 & 42,3 & 58 & 27,2 & 0,044 \\
\hline Mais de 5 agrotóxicos & 75 & 26,6 & 150 & 53,2 & 57 & 20,2 & \\
\hline \multicolumn{8}{|l|}{ Orientação técnica para aquisição de agrotóxicos } \\
\hline Não é responsável pela compra de agrotóxicos & 42 & 24,7 & 72 & 42,4 & 56 & 32,9 & $<0,001$ \\
\hline Compra sem orientação técnica & 42 & 22,6 & 90 & 48,4 & 54 & 29,0 & \\
\hline Compra com orientação técnica & 68 & 38,6 & 97 & 55,1 & 11 & 6,3 & \\
\hline \multicolumn{8}{|l|}{ Equipamento de aplicação } \\
\hline Não-mecanizado & 73 & 24,3 & 150 & 50,0 & 77 & 25,7 & 0,002 \\
\hline Mecanizado & 36 & 35,3 & 51 & 50,0 & 15 & 14,7 & \\
\hline Mecanizado e Não-mecanizado & 42 & 40,0 & 51 & 48,6 & 12 & 11,4 & \\
\hline \multicolumn{8}{|l|}{ Conduta após utilizar agrotóxicos } \\
\hline Toma banho ou se lava imediatamente & 147 & 30,7 & 235 & 49,1 & 97 & 20,3 & $<0,001$ \\
\hline Não toma banho ou não se lava imediatamente & 5 & 10,4 & 22 & 45,8 & 21 & 43,8 & \\
\hline \multicolumn{8}{|l|}{ Realiza leitura do rótulo dos agrotóxicos } \\
\hline Sim & 103 & 39,9 & 123 & 47,7 & 32 & 12,4 & $<0,001$ \\
\hline Não & 43 & 17,4 & 120 & 48,6 & 84 & 34,0 & \\
\hline \multicolumn{8}{|l|}{ Respeita o tempo de carência para colheita } \\
\hline Sim & 111 & 34,4 & 162 & 50,2 & 50 & 15,5 & $<0,001$ \\
\hline Não & 33 & 18,3 & 88 & 48,9 & 59 & 32,8 & \\
\hline \multicolumn{8}{|l|}{ Respeita o tempo de carência para reaplicação } \\
\hline Sim & 119 & 35,7 & 160 & 48,0 & 54 & 16,2 & $<0,001$ \\
\hline Não & 21 & 13,3 & 84 & 53,2 & 53 & 33,5 & \\
\hline \multicolumn{8}{|l|}{$\begin{array}{l}\text { Respeita o tempo de reentrada na lavoura após a } \\
\text { aplicação }\end{array}$} \\
\hline Sim & 116 & 42,5 & 124 & 45,4 & 33 & 12,1 & $<0,001$ \\
\hline Não & 32 & 13,6 & 126 & 53,6 & 77 & 32,8 & \\
\hline
\end{tabular}

*EPI: equipamento de proteção individual. ****Baseada no Critério de Classificação Econômica Brasil (Abep). 
Tabela 5 Ingredientes ativos mais utilizados pelos agricultores do município de Santa Maria de Jetibá/ES considerando as marcas comerciais citadas

\begin{tabular}{|c|c|c|c|}
\hline Ingrediente ativo* & Grupo químico & $n^{\text {**** }}$ & $\%$ 米* \\
\hline Glifosato-sal de di-amônia & Glicina substituída & 339 & 66,4 \\
\hline Mancozebe & Alquilenobis(ditiocarbamato) & 298 & 58,4 \\
\hline Dicloreto de paraquate & Bipiridílio & 296 & 58,0 \\
\hline Deltametrina & Piretróide & 260 & 51,0 \\
\hline Abamectina & Avermectina & 242 & 47,5 \\
\hline Azoxistrobina & Estrobilurina & 203 & 39,8 \\
\hline Difenoconazol & Triazol & 193 & 37,8 \\
\hline Tiametoxam & Neonicotinóide & 187 & 36,7 \\
\hline Lambda-cialotrina & Piretróide & 183 & 35,9 \\
\hline Metalaxil-M & Acilalaninato & 153 & 30,0 \\
\hline Tiofanato-metílico & Benzimidazol & 148 & 29,0 \\
\hline Clorfenapir & Análogo de pirazol & 134 & 26,3 \\
\hline Tebuconazol & Triazol & 126 & 24,7 \\
\hline Imidacloprido & Neonicotinóide & 120 & 23,5 \\
\hline Trifloxistrobina & Estrobilurina & 110 & 21,6 \\
\hline Clorantraniliprole & Antranilamida & 104 & 20,4 \\
\hline Profenofós & Organofosforado & 104 & 20,4 \\
\hline Indoxacarbe & Oxadiazina & 102 & 20,0 \\
\hline Metomil & Metilcarbamato de oxima & 101 & 19,8 \\
\hline Metolacloro & Cloroacetanilida & 98 & 19,2 \\
\hline
\end{tabular}

*Dos 77 Ingredientes ativos (IA) identificados no estudo, foram apresentados nesta tabela os dados dos 20 mais frequentes. **** Corresponde ao número de agricultores que referiram utilizar pelo menos 1 agrotóxico com o respectivo ingrediente ativo. ${ }^{*}$ C***: Calculado levando-se em consideração o total de 510 agricultores que souberam informar a marca comercial dos agrotóxicos utilizados.

\section{Discussão}

Este estudo de base populacional realizado com amostra ampla e representativa, selecionada de modo estratificado e aleatório, com pequenas proporções de perdas, possibilita a extrapolação dos resultados para a população-alvo, composta por pequenos agricultores, cujas práticas agrícolas caracterizam-se pelo predomínio da policultura e utilização de agrotóxicos. Por meio desta investigação, foi possível evidenciar importantes achados que corroboram o perfil de exposição ocupacional aos agrotóxicos encontrados em outras regiões brasileiras, com destaque para a exposição a uma vasta gama de produtos, de elevada toxicidade, por vários anos e sem utilização dos equipamentos de proteção individual recomendados.

Segundo o relatório anual de comercialização de agrotóxicos do Instituto Brasileiro do Meio Ambiente (Ibama) $^{3}$, o Brasil passou de 162.461,96 toneladas de agrotóxicos comercializadas em 2000 para $551.313,25$ em 2016, o que representou um aumento aproximado de $240 \%$. Esse crescimento, no entanto, não foi acompanhado pelo aumento proporcional das áreas cultivadas, que cresceram apenas $49 \%$ no período $^{3,9}$. No Espírito Santo essas diferenças foram ainda maiores: enquanto a comercialização de agrotóxicos aumentou $64,6 \%$ de 2000 a 2016 , a área plantada ou destinada à colheita reduziu em $17 \% \%^{3,9}$.

Do volume total de agrotóxicos comercializados no Brasil em 2016, 58,5\% eram herbicidas, 13,3\% fungicidas e $8,53 \%$ inseticidas ${ }^{9}$, o que parece refletir na tendência de consumo identificada neste estudo. Pesquisa anterior realizada na região de Alto Santa Maria, ES, também revelou perfil de consumo semelhante ${ }^{10}$. Um quadro preocupante é delineado quando se constata o aumento crescente da utilização de fungicidas nas plantações de hortaliças do Brasil. Estima-se que cerca de $20 \%$ da comercialização nacional de fungicidas seja destinada a esse tipo de cultura, na qual o consumo de agrotóxicos por hectare pode chegar a ser 8 a 16 vezes maior quando comparado ao utilizado na cultura da soja, por exemplo ${ }^{11}$.

No Brasil, o Glifosato e seus sais ocuparam o primeiro lugar no ranking dos mais vendidos em 2016. 
Entre os 10 agrotóxicos mais comercializados também estão o Mancozebe e o Dicloreto de Paraquate ${ }^{3}$, corroborando dados encontrados em Santa Maria de Jetibá. Cabe destacar que dentre os 77 ingredientes ativos de utilização frequente pelos agricultores desta investigação, aproximadamente $20 \%$ (n = 15) são proibidos na União Europeia ${ }^{12}$, entre eles Paraquate, Clorfenapir e Profenofós, e aproximadamente 12\% (n $=9$ ) não são registrados nos Estados Unidos ${ }^{13}$.

Diferente do encontrado por outros autores ${ }^{14}$, o grupo químico dos organofosforados não foi amplamente citado neste estudo. Uma das hipóteses pode estar relacionada ao tipo de cultura predominante na região de Santa Maria de Jetibá. Apesar dos organofosforados serem registrados para algumas culturas de hortaliças, também são utilizados em culturas de algodão, soja, trigo ${ }^{8}$, que não são cultivos características na região.

Embora os efeitos à saúde pela exposição aos agrotóxicos não tenham sido objeto desta pesquisa, diversos estudos na literatura associam efeitos potencialmente danosos relacionados com várias substâncias utilizadas pelos agricultores investigados. Segundo dados da Pesticide Action Network (PAN) ${ }^{15}$, $30 \%$ dos ingredientes ativos de uso contínuo pelos agricultores deste estudo $(n=23)$ são classificados como carcinogênicos para humanos ou animais, $17 \%$ ( $n=13$ ) apresentam elevada toxicidade para o sistema reprodutor e $26 \%(n=20)$ são suspeitos de causar alterações no sistema endócrino.

Estudo de Koifman, Koifman e Meyer ${ }^{16}$ demonstrou que em alguns estados brasileiros houve correlação entre a utilização de agrotóxicos e manifestações endócrinas na população exposta. Seus efeitos podem ser decorrentes de sua capacidade de agirem como disruptores endócrinos (DE), substâncias químicas capazes de interferir no funcionamento normal de hormônios ou enzimas de animais e humanos. Glifosato, Mancozebe, Deltrametrina, Tebuconazol, Metalocloro são exemplos de agrotóxicos que apresentam indícios de interação com o sistema endócrino ${ }^{17}$. Goldner et al. ${ }^{18}$ encontraram associação entre o hipotireoidismo e o uso do inseticida organoclorado Clordano, o herbicida Paraquate e os fungicidas Benomil e Mancozebe , tendo sido este último o único associado tanto ao hipertireoidismo como ao hipotireoidismo. Efeitos sobre o sistema reprodutor também foram observados por Hossain, Ali, D’Souza e Nain ${ }^{19}$, que identificaram risco 3 a 9 vezes maior de parâmetros seminais anormais em indivíduos expostos à agrotóxicos.

As evidências em estudos da literatura também indicam possíveis relações entre exposição a agrotóxicos e doenças respiratórias ${ }^{20}$. Organofosforados, Piretróides e Dicloreto de Paraquate foram associados à rinite alérgica no estudo de Koureas e colaboradores ${ }^{21}$.
Associações com desenvolvimento de doenças de Parkinson $^{22}$, linfoma não Hodgkin ${ }^{23}$ e leucemia ${ }^{24}$ permanecem controversas. O herbicida Glifosato, conhecido pela marca Roundup ${ }^{\circledR}$, agrotóxico mais citado neste estudo, também tem sido associado a efeitos negativos sobre a saúde ${ }^{25}$, incluindo provável potencial carcinogênico, de acordo com a International Agency for Research on Cancer $^{26}$. Cabe destacar recente decisão judicial que condenou a empresa Monsanto, fabricante do referido produto, a pagar 289 milhões de dólares em indenizações por suposta associação do glifosato com o desenvolvimento de câncer em trabalhador. Além disso, a empresa responde a mais de cinco mil processos semelhantes nos Estados Unidos ${ }^{27}$.

Também chama a atenção no presente estudo a utilização concomitante de diversos tipos de agrotóxicos, dados que reforçam a exposição ocupacional múltipla a que o agricultor está submetido. Segundo Harding et al. ${ }^{28}$ a exposição simultânea dificulta a investigação dos riscos para a saúde associados à utilização de agrotóxicos, uma vez que os produtos possuem diferentes modos de ação. Conforme destaca Faria ${ }^{29}$, essa investigação torna-se ainda mais complexa considerando que a maior parte das informações disponíveis sobre os efeitos à saúde está concentrada em poucos tipos químicos, não abrangendo a grande variedade de agrotóxicos de uso rotineiro que se tem evidenciado.

Convém destacar o elevado número de agricultores deste estudo que referiu tempo de exposição ocupacional a agrotóxicos superior a 20 anos. Sabe-se que a duração, a frequência e a intensidade da exposição são importantes fatores de risco para o desenvolvimento de efeitos crônicos sobre a saúde ${ }^{30}$. Esses efeitos, no entanto, não têm sido adequadamente caracterizados, pois em geral, tornam-se aparentes apenas após anos de exposição, dependem da toxicidade de seus componentes e de como estes interagem entre $\mathrm{si}^{31}$. Por isso, para compreensão do real efeito desses produtos sobre a saúde humana, torna-se fundamental a identificação dos efeitos sinérgicos de misturas de dois, três ou mais complexas de agrotóxicos, comumente realizada no campo.

A forma de pulverização adotada também aumenta a exposição do agricultor aos agrotóxicos, elevando a probabilidade de ocorrerem efeitos nocivos. Na população estudada, aproximadamente $60 \%$ dos agricultores utilizavam meios não mecanizados (bombas costais manuais) para pulverização dos produtos. Além do maior risco de intoxicação quando comparado aos meios mecanizados, os equipamentos manuais são de uso mais frequente, principalmente entre os pequenos produtores, em função do seu menor custo de aquisição e manutenção ${ }^{32}$.

Nesse cenário de múltiplos riscos, a utilização de EPIs é de fundamental importância, mas, conforme 
verificado em Santa Maria de Jetibá e em estudos semelhantes ${ }^{2,6}$, a adesão dos trabalhadores rurais a esses equipamentos é pequena, fato que aumenta a sua vulnerabilidade ante os efeitos nocivos do uso de agrotóxicos ${ }^{33}$. Neste estudo, a não utilização de EPI foi associada a diversos fatores, dentre eles, sociodemográficos e econômicos. Dos indivíduos que referiram não utilizar EPI, a maioria era do sexo feminino, possuía baixa escolaridade ou pertencia às classes socioeconômicas D ou E. Corroborando a literatura, para Naidoo e colaboradores $^{34}$, nos locais de precárias condições econômicas, os agricultores dariam preferência às necessidades básicas, como alimentos, roupas e transporte, não à aquisição de equipamentos de segurança.

Cabe destacar que, apesar da associação com a classe socioeconômica e o uso de EPIs, o desconforto, a exemplo de outras regiões ${ }^{2}$, foi o fator mais citado pelos agricultores. A forma como esses equipamentos são projetados e os materiais utilizados em sua confecção afetam os mecanismos fisiológicos de termorregulação ${ }^{35}$. Tais fatores, agravados pela intensa exposição solar e elevado esforço físico decorrentes do trabalho no campo, acarretam consequente elevação de temperatura corporal do trabalhador, tornando a utilização dos EPIs incômoda e com riscos de causar danos à saúde decorrentes do estresse térmico ${ }^{35}$. Apesar do grande avanço tecnológico das indústrias, essa limitação ainda não foi superada, tornando fundamental o desenvolvimento de novas tecnologias para confecção de equipamentos de segurança que ofereçam a proteção ao trabalhador ao mesmo tempo que garantam o conforto térmico necessário às atividades agrícolas ${ }^{29}$.

A não utilização de EPIs também foi associada à adoção de outras condutas inseguras no manuseio dos agrotóxicos, como o não cumprimento do tempo de reentrada e tempo de carência para reaplicação ou colheita, não tomar banho ou se lavar imediatamente após contato com esses produtos e não realizar a leitura dos rótulos dos agrotóxicos utilizados. Deve-se destacar que a baixa escolaridade dos agricultores dificulta a leitura e compreensão das informações descritas nos rótulos dos produtos ${ }^{6} \mathrm{e}$ o consequente acesso às informações sobre os riscos associados ao uso de agrotóxicos, o que, por sua vez, pode ter sido determinante para o não seguimento das práticas de segurança recomendadas.

Também se deve evidenciar que a maior parte dos agricultores que não utilizavam EPIs referiu não receber suporte técnico especializado para aquisição dos agrotóxicos, assunto pouco referido neste estudo e em estudo anterior realizado na região ${ }^{10}$. Mais de $30 \%$ dos agricultores informaram comprar agrotóxicos baseados na indicação de vizinhos, amigos, familiares, por conta própria ou por orientação dos vendedores, por intermédio de receituários agronômicos emitidos nas próprias lojas de produtos agropecuários. Em estudo conduzido no Piaú, 87,4\% dos entrevistados referiram comprar agrotóxicos em casas agropecuárias e 92\% afirmaram que nunca precisaram do receituário agronômico para adquiri-los ${ }^{6}$. Na tentativa de aumentar o rigor na emissão dos receituários, essa forma de assistência, denominada "assistência técnica de balcão", tem sido restringida em alguns estados brasileiros, onde se tem exigido que o receituário agronômico seja elaborado por profissionais qualificados apenas após visita in loco nas propriedades ${ }^{36}$.

A associação entre a adoção de práticas inseguras, como a não utilização de EPIs, e fatores sociais e econômicos, como escolaridade, classe socioeconômica e falta de suporte técnico para aquisição de agrotóxicos, demonstra que o agricultor não deve ser o único responsabilizado pelas atitudes que colocam em risco sua saúde, como frequentemente é observado ${ }^{37}$. Ao contrário, revelam a necessidade de desenvolvimento de políticas públicas que assegurem e estimulem maior acesso à educação, renda e assistência agrícola especializada, como forma de garantir maior proteção à saúde do trabalhador rural.

Segundo Veiga ${ }^{38}$, o papel do agrotóxico pode ser analisado tanto do ponto de vista do seu risco potencial à saúde humana e ao meio ambiente, quanto do ponto de vista do seu papel de agente necessário e catalisador do processo produtivo rural. No entanto, tem-se feito muito esforço para promover o uso de agrotóxicos a fim de aumentar a produtividade e pouco voltado para a proteção da saúde e do meio ambiente ${ }^{39}$. Esse modelo de desenvolvimento vem gerando impactos sociais e ambientais de curto, médio e longo prazo que têm sido custeados por toda a população, decorrente de gastos públicos para recuperação de áreas contaminadas, prevenção, diagnóstico e tratamento das intoxicações agudas e crônicas, afastamentos e aposentadorias por invalidez e mortes de trabalhadores rurais, sem que haja coparticipação das indústrias químicas na socialização destes custos, muitas vezes irreparáveis ${ }^{1}$.

Essa problemática traz à tona a necessidade de encontrar soluções que tenham como objetivo a utilização de agrotóxicos com vistas à minimização dos impactos sobre a saúde humana e à sustentabilidade ambiental. Esse desafio se reveste de alta complexidade pelo caráter multidimensional da temática, pelos diferentes interesses dos setores envolvidos e pelas controversas visões sobre benefícios e malefícios que o uso dos agrotóxicos produz ${ }^{40}$.

Diversas são as estratégias de enfrentamento da utilização desordenada de agrotóxicos no Brasil. Entre elas, cabe destacar: o fortalecimento dos Núcleos de Vigilância de Saúde de Populações Expostas a Agrotóxicos (VSPEA) e das políticas públicas que 
visem o incentivo ao uso de tecnologias alternativas de produção ${ }^{41}$, a implementação de ações educativas efetivas para promoção do uso seguro dos agrotóxicos, o subsídio para aquisição de EPIs, a taxação sobre a comercialização dos agrotóxicos e a implantação de subsídios para produtores que utilizem manejo integrado de pragas e práticas de cultivo mais agroecológi$\operatorname{cas}^{40}$. Além disso, torna-se fundamental a qualificação das equipes de saúde para melhorar o diagnóstico, o manejo e a notificação dos casos de intoxicação, a implementação de indicadores biológicos alternativos para monitoramento e diagnóstico dos casos de intoxicação e o desenvolvimento de EPIs com materiais que, além de ser eficazes, apresentem conforto térmico e custos acessíveis ${ }^{29}$.

\section{Considerações finais}

Este estudo confirma a severidade da exposição ocupacional aos agrotóxicos a que estão submetidos os agricultores de Santa Maria de Jetibá, uma população de trabalhadores rurais exposta de forma prolongada a múltiplos agrotóxicos, em sua maioria de elevada toxidade. Além disso, foram observadas práticas inseguras de manuseio, como a não utilização de equipamentos de proteção individual e o desrespeito aos tempos de reentrada e de carência para reaplicação e colheita.

Compreender as atividades adotadas no campo e as lacunas de conhecimento que levam às práticas inseguras é fundamental para o planejamento de ações de vigilância em saúde das populações expostas a agrotóxicos, como forma de minimizar os impactos desta exposição em curto, médio e longo prazo sobre a saúde do agricultor. Aliado às ações de vigilância, deve-se buscar constantemente o desenvolvimento de novas metodologias agroecológicas de produção, com vistas à redução do consumo exagerado de agrotóxicos que têm gerado impactos incalculáveis e irrecuperáveis sobre a saúde do ser humano e do meio ambiente.

\section{Contribuição de autoria}

Todos os autores declaram que participaram diretamente no planejamento, elaboração do estudo, revisão bibliográfica, análise de dados, redação do artigo e aprovação da versão final publicada e se responsabilizam publicamente pelo seu conteúdo.

\section{Referências}

1. Brasil. Ministério da Saúde. Secretaria de Vigilância em Saúde. Departamento de Vigilância em Saúde Ambiental e Saúde do Trabalhador. Relatório Nacional de Vigilância em Saúde de Populações Expostas a Agrotóxicos. Brasília; 2016.

2. Jallow MF, Awadh DG, Albaho MS, Devi VY, Thomas BM. Pesticide knowledge and safety practices among farm workers in Kuwait: results of a survey. Int J Environ Res Public Health. 2017;14(4):340.

3. Instituto Brasileiro do Meio Ambiente e dos Recursos Naturais Renováveis. Relatórios de comercialização de agrotóxicos [Internet]. Brasília, DF, 2019 [citado em 20 fev 2018]. Disponível em: http://www.ibama.gov.br/agrotoxicos/relatorios-decomercializacao-de-agrotoxicos

4. Miranda AC, Moreira JC, de Carvalho R, Peres F. Neoliberalism, pesticide consumption and food sovereignty crisis in Brazil. Cien Saude Colet. 2007;12(1):7-14.

5. Pignati WA, Lima F, Lara SS, Correa MLM, Barbosa JR, Leão L, et al. Spatial distribution of pesticide use in Brazil: a strategy for health surveillance. Cien Saude Colet. 2017;22(10):3281-93.
6. Santana CM, Costa AR, Nunes RMP, Nunes NMF, Peron AP, Melo-Cavalcante AAC, et al. Exposição ocupacional de trabalhadores rurais a agrotóxicos. Cad Saude Colet. 2016;24(3):301-7.

7. Associação Brasileira de Empresas de Pesquisa. Critério de Classificação Econômica Brasil 2014 [Internet]. São Paulo; 2013 [citado em 29 nov 2015]. Disponível em: http://www.abep.org/ criterio-brasil

8. Brasil. Ministério da Agricultura, Pecuária e Abastecimento. Sistema de Agrotóxicos Fitossanitários [Internet]. Brasília; c2003 [citado em 15 jun 2017]. Disponível em: http://agrofit. agricultura.gov.br/agrofit_cons/principal_agrofit_cons

9. Instituto Brasileiro de Geografia e Estatística. Sistema IBGE de Recuperação Automática: produção agrícola municipal [Internet]. Rio de Janeiro; 2019 [citado em 10 fev 2018]. Disponível em: https://sidra.ibge.gov.br/tabela/5457

10. Jacobson LS, Hacon SS, Alvarenga L, Goldstein RA, Gums C, Buss DF, et al. Comunidade pomerana e uso de agrotóxicos: uma realidade pouco conhecida. Cien Saude Colet. 2009;14(6):2239-49. 
11. Carneiro FF, Rigotto RM, Augusto LGS, Friedrich K, Búrigo AC, organizadores. Dossiê Abrasco: um alerta sobre os impactos dos agrotóxicos na saúde. Rio de Janeiro: EPSJV; 2015.

12. European Commission. Plants: EU Pesticides Database [Internet]. Brussels; 2016 [citado em 12 mar 2018]. Disponível em: http:// ec.europa.eu/food/plant/pesticides/eupesticides-database/public/?event=product. selection\&language $=\mathrm{EN} 2018$

13. United States Environmental Protection Agency. Search by Product [Internet]. Washington, DC; 2018 [citado em 15 mar 2018]. Disponível em: https://iaspub.epa.gov/apex/pesticides/ $\mathrm{f} ? \mathrm{p}=\mathrm{PPLS}: 5::: \mathrm{NO}$

14. Faria NMX, Rosa JARD, Facchini LA. Intoxicações por agrotóxicos entre trabalhadores rurais de fruticultura, Bento Gonçalves, RS. Rev Saude Publica. 2009;43(2):335-44.

15. Pesticide Action Network. Pesticides Database: pesticide products [Internet]. Berkeley; c2019 [citado em 25 mar 2018]. Disponível em: http:// www.pesticideinfo.org/Search_Products. jsp\#ProdSearch

16. Koifman S, Koifman RJ, Meyer A. Human reproductive system disturbances and pesticide exposure in Brazil. Cad Saude Pub. 2002;18(2):435-45.

17. Mnif W, Hassine AI, Bouaziz A, Bartegi A, Thomas O, Roig B. Effect of endocrine disruptor pesticides: a review. Int J Environ Res Public Health. 2011;8(6):2265-303.

18. Goldner WS, Sandler DP, Yu F, Hoppin JA, Kamel F, Levan TD. Pesticide use and thyroid disease among women in the Agricultural Health Study. Am J Epidemiol. 2010;171(4):455-64.

19. Hossain F, Ali O, D’Souza UJ, Naing DK. Effects of pesticide use on semen quality among farmers in rural areas of Sabah, Malaysia. J Occup Health. 2010;52(6):353-60.

20. Cherry N, Beach J, Senthilselvan A, Burstyn I. Pesticide use and asthma in Alberta grain farmers. Int J Environ Res Public Health. 2018;15(3):526.

21. Koureas M, Rachiotis G, Tsakalof A, Hadjichristodoulou C. Increased frequency of rheumatoid arthritis and allergic rhinitis among pesticide sprayers and associations with pesticide use. Int J Environ Res Public Health. 2017;14(8):865

22. Breckenridge CB, Berry C, Chang ET, Sielken RL Jr, Mandel JS. Association between Parkinson's disease and cigarette smoking, rural living, wellwater consumption, farming and pesticide use: systematic review and meta-analysis. PLoS One. 2016;11(4):e0151841.

23. Schinasi L, Leon ME. Non-Hodgkin lymphoma and occupational exposure to agricultural pesticide chemical groups and active ingredients: a systematic review and meta-analysis. Int J Environ Res Public Health. 2014;11(4):4449-527.

24. Lerro CC, Andreotti G, Koutros S, Lee WJ, Hofmann JN, Sandler DP, et al. Alachlor use and cancer incidence in the agricultural health study: an updated analysis. J Natl Cancer Inst. 2018;110(9):950-8.

25. Cai W, Ji Y, Song X, Guo H, Han L, Zhang F, et al. Effects of glyphosate exposure on sperm concentration in rodents: a systematic review and meta-analysis. Environ Toxicol Pharmacol. 2017;55:148-55.

26. International Agency for Research on Cancer. IARC Monographs on the Evaluation of Carcinogenic Risks to Humans. Lyon; 2018 [citado em 6 out 2018]. Disponível em: https://monographs.iarc.fr/ list-of-classifications

27. Monsanto ordered to pay $\$ 289$ million in roundup cancer trial. The New York Times [Internet]. 10 ago 2018 [citado em 6 out 2018]. Disponível em: https://www.nytimes.com/2018/08/10/business/ monsanto-roundup-cancer-trial.html

28. Harding AH, Fox D, Chen Y, Pearce N, Fishwick $D$, Frost G. Prospective Investigation of Pesticide Applicators' Health (PIPAH) study: a cohort study of professional pesticide users in Great Britain. BMJ Open. 2017;7(10):e018212.

29. Faria NMX. Modelo de desenvolvimento, agrotóxicos e saúde: prioridades para uma agenda de pesquisa e ação. Rev Bras Saude Ocup. 2012;37(125):31-9.

30. Lu JL. Assessment of Pesticide-related pollution and occupational health of vegetable farmers in Benguet Province, Philippines. J Health Pollut. 2017;7(16):49-57.

31. Soares W, Almeida RMVR, Moro S. Trabalho rural e fatores de risco associados ao regime de uso de agrotóxicos em Minas Gerais, Brasil. Cad Saude Publica. 2003;19(4):1117-27.

32. Detófano D, Teixeira ML, Oliveira LFSd, Fuentefria AM. Evaluation of toxicity risks in farmers exposed to pesticides in an agricultural community in Concórdia, Santa Catarina State, Brazil. Acta Sci Health Sci. 2013; 35(1):111-8.

33. Bedor CNG, Ramos LO, Pereira PJ, Rêgo MAV, Pavão AC, Augusto LGS. Vulnerabilidades e situações de riscos relacionados ao uso de agrotóxicos na fruticultura irrigada. Rev Bras Epidemiol. 2009;12(1):39-49.

34. Naidoo S, London L, Burdorf A, Naidoo RN, Kromhout H. Agricultural activities, pesticide use and occupational hazards among women working in small scale farming in Northern KwaZuluNatal, South Africa. Int J Occup Environ Health. 2008;14(3):218-24.

35. Veiga MM, Almeida R, Duarte F. O desconforto térmico provocado pelos equipamentos de proteção individual (EPI) utilizados na aplicação de agrotóxicos. Laboreal. 2016;12(2):83-94.

36. Conselho Regional de Engenharia e Agronomia do Rio Grande do Sul (BR). Câmara Especializada de Agronomia. Norma de fiscalização $\mathrm{n}^{\circ} 2$, de 2015. Porto Alegre; 2015 [citado em 15 abr 2018]. 
Disponível em: http://www.crea-rs.org.br/site/ documentos/NORMA\%2002-15(1).pdf

37. Rigotto, R. Agrotóxicos, trabalho e saúde: vulnerabilidade e resistência no contexto da modernização agrícola no baixo Jaguaribe/CE. Fortaleza: Edições UFC; 2011.

38. Veiga MM. Agrotóxicos: eficiência econômica e injustiça socioambiental. Cien Saude Colet. 2007;12(1):145-52.

39. Mrema EJ, Ngowi AV, Kishinhi SS, Mamuya SH. Pesticide exposure and health problems among female horticulture workers in Tanzania. Environ Health Insights. 2017;11:1-13.

40. Waichman AV. A problemática do uso de agrotóxicos no Brasil: a necessidade de construção de uma visão compartilhada por todos os atores sociais. Rev Bras Saude Ocup. 2012;37(125):42-7.

41. Brasil. Ministério da Saúde. Coordenação Geral de Vigilância em Saúde Ambiental. Documento orientador para a implementação da vigilância em saúde de populações expostas a agrotóxicos. Brasília, DF; 2012. 